УДК 532.5.013

\title{
D'Alembert's Paradox in Near Real Conditions
}

\author{
Alexander V. Koptev* \\ Institute of Water Transport \\ Admiral Makarov State University of Maritime and Inland Shipping \\ Dvinskaya, 5/7, Saint-Petersburg, 198035
}

Russia

Received 09.04.2016, received in revised form 08.12.2016, accepted 20.01.2017

This paper examines the D'Alembert's paradox in the flow of ideal incompressible medium around a cylinder when flow conditions are close to real ones. The velocity profile of incoming flow is specified on a section at a finite distance from the cylinder. An additional parameter is introduced to determine the degree of asymmetry of the incoming flow. Initially, the value of this parameter is assumed to be small. The parameter that determines the geometric dimensions of the cylinder is also introduced. Some cases are identified when the situation is close to D'Alembert's paradox in its classic version, and when it is not. It depends on the values of introduced parameters.

Keywords: ideal incompressible medium, flow around a cylinder, Euler equations, integral, velocity profile, asymmetrical flow, lift, drag.

DOI: 10.17516/1997-1397-2017-10-2-170-180.

\section{Introduction}

D'Alembert's paradox is a well-known phenomenon in fluid mechanics. Consider a continuous unbounded steady flow of an ideal (inviscid) incompressible fluid around a body when flow parameters are equalized far ahead and behind the body. The paradox lies in the fact that the drag calculated on the basis of equations of motion vanishes.

For the flow around a cylinder, this phenomenon was discovered by French mathematician D'Alembert in 1744. For the flow around the body of an arbitrary shape similar result was obtained by L. Euler in 1745 .

By virtue of the Galilean principle of relativity one can assume that similar phenomenon exists when a body moves with constant velocity in an unbounded volume of an ideal incompressible fluid that is at rest at infinity. In this case the drag calculated on the basis of equations of motion is equal to zero as well.

This theoretical result is rather strange from the point of view of the theory and common sense. Numerous experiments argued against this phenomenon. When a body moves in a continuous medium there is non-zero resistance force. So the phenomenon, on which the attention of D'Alembert was drawn, is not supported by experimental results. The term "paradox" was first used in relation to this phenomenon by D'Alembert in 1768. As a result, this phenomenon received the name D'Alembert's paradox. There are also other names: the Euler paradox or the paradox of D'Alembert-Euler.

\footnotetext{
*Alex.Koptev@mail.ru

(c) Siberian Federal University. All rights reserved
} 
The phenomenon was considered in many studies [1-6]. Conditions of problem formulation that lead to the paradox were found in these papers. The following factors are indicated among others: setting velocity at infinity, infinite domain, symmetry of incoming flow, stationary motion and non-zero viscosity.

However, it is not clear whether this phenomenon occurs in reality if the assumptions are slightly changed. It is almost impossible to create a symmetrical steady flow of infinite extent. In addition, the motion of any real flow always occurs in the presence of gravity. So, in practice there are always some deviations from those idealized conditions that originally are assumed when dealing with this phenomenon. The question arises whether the D'Alembert's paradox would occur in more realistic conditions. If the drag is not equal to zero it is important to determine the value of its deviation from zero.

The study of the D'Alembert's paradox under more realistic conditions is the purpose of the paper.

\section{Problem Statement}

To study the D'Alembert's paradox it is necessary to calculate the drag resulting in the flow of an ideal incompressible medium around a body. In addition, it is suggested to calculate the lift because it is convenient to determine the ratio of drag to lift. Initially we need to solve the flow problem and determine flow velocities and pressure.

Let us consider an ideal incompressible medium. We assume that there is the stationary flow without separation around a circular cylinder of radius $R$. We change some of the conditions wherein the D'Alembert's paradox is usually considered [3-6]. The velocity of the incoming flow is not set at infinity but at some finite distance $L$ from the cylinder. Thus, together with the radius $R$, additional parameter $L$ is introduced which determines the location where initial velocity profile is defined. The condition of infinite flow is relaxed.

We assume that the incoming flow is parallel and it has a predetermined velocity profile in the section located at a distance $L$ from the body. Moreover, the velocity profile is assumed to be slightly asymmetrical. Then the condition of the absolute symmetry of the incoming flow is also relaxed.

We propose to take into account the effect of gravity. This is not a resistance force but it contributes to the vertical component of the force acting on the streamline body. This means that it affects the ratio between horizontal and vertical components of the forces acting on the body so in the analysis of the D'Alembert's paradox under real conditions the force of gravity must be taken into account.

By this means three conditions assumed in the classical formulation are significantly changed. The obtained formulation is much closer to the real conditions.

Let us consider two dimensional problem and introduce the Cartesian coordinate system $X O Y$. The origin of the coordinate system is on the cylinder axis.

Let us consider the classical Euler equations for $2 D$ steady-state motion of an ideal (nonviscous) incompressible medium. For the case when the external mass force is the gravity these equations are

$$
\begin{gathered}
u \frac{\partial u}{\partial x}+v \frac{\partial u}{\partial y}=-\frac{\partial p}{\partial x} \\
u \frac{\partial v}{\partial x}+v \frac{\partial v}{\partial y}=-\frac{\partial p}{\partial y}-g \\
-171-
\end{gathered}
$$




$$
\frac{\partial u}{\partial x}+\frac{\partial v}{\partial y}=0
$$

Let us introduce dimensionless variables. We assume that $L$ is the length scale, $U_{0}$ is the velocity scale, $\rho U_{0}^{2}$ is the pressure scale and $\frac{U_{0}^{2}}{L}$ is the scale of acceleration. All dimensionless variables have the same notation as the corresponding dimensional variables.

In the chosen coordinate system the streamline body is given by the equation $x^{2}+y^{2}=r^{2}$, where $r=\frac{R}{L}$ and $0<r<1$.

The major unknowns are the longitudinal and transverse velocities $u, v$ and pressure $p$. We also introduce the stream function $\Psi_{1}(x, y)[1-3]$. Then

$$
u=\frac{\partial \Psi_{1}}{\partial y}, \quad v=-\frac{\partial \Psi_{1}}{\partial x} .
$$

Solution is constructed in the form of power expansion

$$
\Psi_{1}(x, y)=\sum_{n=0}^{N} \sum_{m=0}^{N-n} a_{n m} x^{n} y^{m}
$$

where $a_{n m}$ are some coefficients and $N$ is the maximum power in the expansion.

Let us consider boundary conditions. The first one is the condition of impermeability on the surface of streamline body [1-3]. In our case, the stream line along the contour of the streamlined body is defined by the equation $x^{2}+y^{2}=r^{2}$ and the impermeability condition has the form

$$
\left.(\vec{U} \cdot \vec{n})\right|_{r^{2}=x^{2}+y^{2}}=0,
$$

where $\vec{U}(u, v)$ is the velocity vector and $\vec{n}\left(\frac{x}{r}, \frac{y}{r}\right)$ is the normal vector to the stream line.

For the second boundary condition we define the velocity profile in cross-section located at a finite distance from the streamlined body. We choose a cross-section parallel to the $O Y$ axis located on the left side from the cylinder at the distance $L$ from the origin. In dimensionless variables such section is determined by equation $x=-1$. We assume that in this section the flow is parallel to the $O X$ axis. Then transverse velocity is zero. The second boundary condition is

$$
v(-1, y)=0 .
$$

To solve the problem it is suggested to use not the Euler equations but the first integral of these equations. The first integral of the Euler equations contains additional information on relationships between variables, and allows us to consider the flow problem and D'Alembert's paradox from another point of view. The first integral in the case of $3 D$ motion of a viscous imcompressible medium is given in [7-11]. In the case of $2 D$ steady-state motion of an ideal imcompressible medium one need to put $\frac{1}{R e}=0, \frac{\partial}{\partial t}=0$ and $\frac{\partial}{\partial z}=0$.

Then the first integral of the Euler equations takes the form

$$
\begin{gathered}
p-p_{0}=-g y-\frac{U^{2}}{2}-d, \\
u^{2}-v^{2}=-\frac{\partial^{2} \Psi_{2}}{\partial x^{2}}+\frac{\partial^{2} \Psi_{2}}{\partial y^{2}}, \\
u v=-\frac{\partial^{2} \Psi_{2}}{\partial x \partial y} . \\
-172-
\end{gathered}
$$


Here $p_{0}$ is an additive pressure constant, $\Psi_{2}$ is new unknown variable due to integration, $U$ is the velocity value and $d$ is the vortex energy. The last is defined by the following expression

$$
d=-\frac{1}{2}\left(\frac{\partial^{2} \Psi_{2}}{\partial x^{2}}+\frac{\partial^{2} \Psi_{2}}{\partial y^{2}}\right)
$$

Equations (8-10) are simpler than original equations (1-2). Derivatives of unknowns $u, v, p$ are absent in $(8-10)$ so it is easier to find the solution of the problem. It is necessary to satisfy continuity equation (3), boundary conditions (6-7) and to solve equations (9-10).

Unknown value of $p$ is determined from (8) and (11). When pressure function $p(x, y)$ is known one can determine drag and lift and begin to study the D'Alembert paradox.

\section{Solution of the flow problem}

Preliminary analysis shows that it is sufficient to set $N=5$ and assume $0 \leqslant n+m \leqslant 5$ in the right-hand side of expression (5).

Then, according to (4), velocities are defined by the fourth degree polynomials. The first term for $u(x, y)$ is $a_{01}$. Let us assume for simplicity that $a_{01}=1$. This means that the first term in the expansion of dimensional longitudinal velocity is equal to $U_{0}$.

Then we have the following expressions for longitudinal and transversal velocities

$$
\begin{aligned}
u= & 1+a_{11} x+2 a_{02} y+a_{21} x^{2}+2 a_{12} x y+3 a_{03} y^{2}+a_{31} x^{3}+2 a_{22} x^{2} y+ \\
& +3 a_{13} x y^{2}+4 a_{04} y^{3}+a_{41} x^{4}+2 a_{32} x^{3} y+3 a_{23} x^{2} y^{2}+4 a_{14} x y^{3}+5 a_{05} y^{4}, \\
v= & -\left(a_{10}+a_{11} y+2 a_{20} x+3 a_{30} x^{2}+2 a_{21} x y+a_{12} y^{2}+4 a_{40} x^{3}+3 a_{31} x^{2} y+\right. \\
& \left.+2 a_{22} x y^{2}+a_{13} y^{3}+5 a_{50} x^{4}+4 a_{41} x^{3} y+3 a_{32} x^{2} y^{2}+2 a_{23} x y^{3}+a_{14} y^{4}\right),
\end{aligned}
$$

where $a_{n m}$ are some coefficients introduced in (5).

Equalities (12-13) satisfy continuity equation (3), and we need to determine coefficients $a_{n m}$.

It is convenient to divide the solution of the flow problem into three stages. In the first stage one need to satisfy boundary conditions $(6-7)$. Then, one should solve equations (9-10) with respect to unknown $\Psi_{2}$. In the last stage one need to determine $p$, according to (8), and calculate the drag and lift.

\subsection{Boundary conditions}

Let us consider relations (12-13). They contain undetermined coefficients $a_{n m}$. These coefficients are treated as basic ones. They are chosen so that boundary conditions (6-7) are satisfied. To satisfy (6) the following relation must be true

$$
\begin{aligned}
u x+v y= & \left(r^{2}-x^{2}-y^{2}\right)\left(\xi_{00}+\xi_{10} x+\xi_{01} y+\xi_{20} x^{2}+\xi_{11} x y+\right. \\
& \left.+\xi_{02} y^{2}+\xi_{30} x^{3}+\xi_{21} x^{2} y+\xi_{12} x y^{2}+\xi_{03} y^{3}\right)
\end{aligned}
$$

where $\xi_{k l}$ are some auxiliary coefficients.

Relation (14) allows us to determine the values of auxiliary coefficients $\xi_{k l}$ in terms of basic ones and to express coefficients $a_{n m}$ with bigger values of $(n, m)$ in terms of coefficients with 
smaller values of $(n, m)$. Coefficients $\xi_{k l}$ depends on $a_{n m}$. They play an auxiliary role and are not used. Upon some rearrangement, we obtain

$$
\begin{aligned}
& a_{50}=-\frac{1}{r^{2}}\left(a_{30}+\frac{a_{10}}{r^{2}}\right), \quad a_{41}=-\frac{1}{r^{2}}\left(a_{21}+\frac{1}{r^{2}}\right), \quad a_{05}=-\frac{1}{r^{2}}\left(a_{03}+\frac{1}{r^{2}}\right), \\
& a_{32}=-\frac{1}{r^{2}}\left(a_{12}+a_{30}+\frac{2 a_{10}}{r^{2}}\right), \quad a_{14}=-\frac{1}{r^{2}}\left(a_{12}+\frac{a_{10}}{r^{2}}\right), \\
& a_{23}=-\frac{1}{r^{2}}\left(a_{21}+a_{03}+\frac{2}{r^{2}}\right), \quad a_{40}=\frac{1}{2}\left(a_{22}+\frac{a_{02}-a_{20}}{r^{2}}\right), \\
& a_{31}=a_{13}=-\frac{a_{11}}{r^{2}}, \quad a_{04}=\frac{1}{2}\left(a_{22}-\frac{a_{02}-a_{20}}{r^{2}}\right) .
\end{aligned}
$$

Taking into account (15), expressions for longitudinal and transversal velocities take the form

$$
\begin{aligned}
u(x, y)=1 & +a_{11} x+2 a_{02} y+a_{21} x^{2}+2 a_{12} x y+3 a_{03} y^{2}-\frac{a_{11} x^{3}}{r^{2}}+2 a_{22} x^{2} y-\frac{3 a_{11} x y^{2}}{r^{2}}+ \\
& +2\left(a_{22}-\frac{a_{02}-a_{20}}{r^{2}}\right) y^{3}-\frac{1}{r^{2}}\left(a_{21}+\frac{1}{r^{2}}\right) x^{4}-\frac{2}{r_{2}}\left(a_{12}+a_{30}+\frac{2 a_{10}}{r^{2}}\right) x^{3} y- \\
& -\frac{3}{r^{2}}\left(a_{21}+a_{03}+\frac{2}{r^{2}}\right) x^{2} y^{2}-\frac{4}{r^{2}}\left(a_{12}+\frac{a_{10}}{r^{2}}\right) x y^{3}-\frac{5}{r^{2}}\left(a_{03}+\frac{1}{r^{2}}\right) y^{4}, \\
v(x, y)= & -a_{10}-2 a_{20} x-a_{11} y-3 a_{30} x^{2}-2 a_{21} x y-a_{12} y^{2}-2\left(a_{22}+\frac{a_{02}-a_{20}}{r^{2}}\right) x^{3}+ \\
& +\frac{3 a_{11} x^{2} y}{r^{2}}-2 a_{22} x y^{2}+\frac{a_{11} y^{3}}{r^{2}}+\frac{5}{r^{2}}\left(a_{30}+\frac{a_{10}}{r^{2}}\right) x^{4}+\frac{4}{r^{2}}\left(a_{21}+\frac{1}{r^{2}}\right) x^{3} y+ \\
& +\frac{3}{r^{2}}\left(a_{12}+a_{30}+\frac{2 a_{10}}{r^{2}}\right) x^{2} y^{2}+\frac{2}{r^{2}}\left(a_{21}+a_{03}+\frac{2}{r^{2}}\right) x y^{3}+\frac{1}{r^{2}}\left(a_{12}+\frac{a_{10}}{r^{2}}\right) y^{4} .
\end{aligned}
$$

Let us impose restrictions on coefficients to satisfy second boundary condition (7). It follows from (7) that for $x=-1$ the transversal velocity should be equal to zero. Then all coefficients at $y^{m}$ in expression for $v(-1, y)$ must be equal to zero. In our case $0 \leqslant m \leqslant 4$ and we have five additional relations

$$
\begin{aligned}
& a_{10}-2 a_{20}+3 a_{30}-2 a_{22}-\frac{2}{r^{2}}\left(a_{02}-a_{20}\right)-\frac{5}{r^{2}}\left(a_{30}+\frac{a_{10}}{r^{2}}\right)=0, \\
& a_{11}-2 a_{21}-\frac{3 a_{11}}{r^{2}}+\frac{4}{r^{2}}\left(a_{21}+\frac{1}{r^{2}}\right)=0, \quad a_{11}-2\left(a_{21}+a_{03}+\frac{2}{r^{2}}\right)=0, \\
& a_{12}-2 a_{22}-\frac{3}{r^{2}}\left(a_{12}+a_{30}+\frac{2 a_{10}}{r^{2}}\right)=0, \quad a_{12}+\frac{a_{10}}{r^{2}}=0 .
\end{aligned}
$$

Let us consider the longitudinal velocity at $x=-1$. We have the following expression

$$
u(-1, y)=1+e_{1} y+e_{2} y^{2}+e_{3} y^{3}+e_{4} y^{4},
$$

where $e_{j}$ are some coefficients.

As it follows from the preceding discussion, this expression must be an asymmetrical function so the odd coefficients are not equal to zero, that is, $e_{1} \neq 0$ and $e_{3} \neq 0$. Then we have two additional relations

$$
\begin{aligned}
& a_{02}-a_{12}+a_{22}+\frac{1}{r^{2}}\left(a_{12}+a_{30}+\frac{2 a_{10}}{r^{2}}\right)=\frac{e_{1}}{2}, \\
& a_{22}-\frac{1}{r^{2}}\left(a_{02}-a_{20}\right)+\frac{2}{r^{2}}\left(a_{12}+\frac{a_{10}}{r^{2}}\right)=\frac{e_{3}}{2},
\end{aligned}
$$

where $e_{1}$ and $e_{3}$ are some non-zero parameters.

If equations (18-19) are satisfied then boundary condition (7) is also satisfied. There are seven 
equations for nine unknown coefficients. Three of them are chosen as basic ones. It is suggested to choose $a_{10}, a_{02}, a_{11}$ as basic coefficients and other coefficients are expressed in terms of basic ones. Then relations (18-19) is the system of seven linear equations for six unknowns $a_{20}, a_{30}$, $a_{21}, a_{12}, a_{03}, a_{22}$. Preliminary analysis shows that solution exists under additional condition

$$
e_{3}=\frac{e_{1}}{1-r^{2}}
$$

If condition (20) holds then the solution of linear system is unique and it has the following form

$$
\begin{aligned}
& a_{12}=-\frac{a_{10}}{r^{2}}, \quad a_{20}=2 a_{10}+a_{02}\left(1+3 r^{2}\right)+\frac{e_{1} r^{2}\left(3 r^{2}-2\right)}{2\left(2-r^{2}\right)}, \\
& a_{03}=-\frac{a_{11}}{2\left(2-r^{2}\right)}+\frac{2\left(r^{2}-1\right)}{r^{2}\left(2-r^{2}\right)}, \quad a_{30}=2 r^{2} a_{02}+\frac{a_{10}\left(r^{2}-1\right)}{r^{2}}-r^{2} e_{1}, \\
& a_{21}=\frac{1}{2\left(2-r^{2}\right)}\left(a_{11}\left(3-r^{2}\right)-\frac{4}{r^{2}}\right), \quad a_{22}=-3 a_{02}-\frac{2 a_{10}}{r^{2}}+\frac{3 e_{1}}{2} .
\end{aligned}
$$

As a result, relations (16-17) and (21) satisfy both continuity equation (3) and boundary conditions (6-7). The first stage of solution is completed.

\subsection{Determination of associate unknown}

Let us consider equations (9-10). These equations must be solved with respect to associate unknown $\Psi_{2}$. In the framework of the present approach $\Psi_{2}$ is also represented in the form of power expansion

$$
\Psi_{2}(x, y)=\sum_{n=0}^{5} \sum_{m=0}^{5-n} b_{n m} x^{n} y^{m},
$$

where $b_{n m}$ are some unknown coefficients, $0 \leqslant n+m \leqslant 5$.

Let us find $u^{2}-v^{2}$ and $u v$. To do this we equate coefficients at the same powers on both sides of the relation. We arrive to a system of linear equations with respect to $b_{n m}$. The system has a solution if certain conditions of compatibility are satisfied. These conditions are

$$
\begin{aligned}
& a_{11} a_{10}+12\left(2-r^{2}\right) a_{02}+\frac{2\left(4-3 r^{2}\right)}{r^{2}} a_{10}-6 e_{1}\left(2-r^{2}\right)=0, \\
& \frac{\left(-r^{4}+2 r^{2}+8\right)}{r^{2}} a_{11} a_{10}+r^{2}\left(13-3 r^{2}\right) a_{11} a_{02}+\frac{4\left(r^{2}-6\right)}{r^{2}} a_{10}+ \\
& \quad+\frac{2\left(3 r^{4}-24 r^{2}+8\right)}{r^{2}} a_{02}+e_{1} r^{2} \frac{\left(-3 r^{4}+22 r^{2}-24\right)}{2\left(2-r^{2}\right)} a_{11}+e_{1} \frac{\left(3 r^{4}-36 r^{2}+44\right)}{\left(2-r^{2}\right)}=0, \\
& \frac{r^{2}}{2\left(2-r^{2}\right)} a_{11}^{2}+12 r^{2} a_{02}^{2}+\frac{4}{r^{2}} a_{10}^{2}+\frac{4\left(3 r^{2}-2\right)}{r^{2}} a_{02} a_{10}- \\
& \quad-\frac{4}{r^{2}\left(2-r^{2}\right)} a_{11}-6 e_{1} r^{2} a_{02}-3 e_{1} \frac{\left(6-r^{2}\right)}{\left(2-r^{2}\right)} a_{10}=0 .
\end{aligned}
$$

Equations (23) are non-linear because Euler equations (1-2) are nonlinear. The solution of (23) defines the values of three base coefficients $a_{10}, a_{11}, a_{02}$. Knowing these coefficients, one can determine all other quantities, namely, $u, v$ and $\Psi_{2}$.

In particular, the coefficients $b_{n m}$ that define $\Psi_{2}$ are the solution of inhomogeneous linear system of equations. Unknown function $\Psi_{2}$ includes three still unknown coefficients $a_{11}, a_{02}, a_{10}$. The second stage of solution is completed. 


\subsection{Determination of pressure, drag and lift}

In the third stage of solution one need to determine $p$ and values of drag and lift. To do this we use expressions (8) and (11). The unknown $p$ is defined by $u, v, \Psi_{2}$, and they depend on base coefficients $a_{11}, a_{02}, a_{10}$.

Within the framework of the used approximation with $N=5$, expression for $p$ has the form

$$
\begin{aligned}
p=-g y+\left(p_{00}+p_{10} x+p_{01} y+p_{20} x^{2}+p_{11} x y+\right. & p_{02} y^{2}+p_{30} x^{3}+ \\
& \left.+p_{21} x^{2} y+p_{12} x y^{2}+p_{03} y^{3}\right),
\end{aligned}
$$

where $p_{n m}$ are some coefficients which also depend on base coefficient $a_{11}, a_{02}, a_{10}$.

Let us now consider drag and lift. Given the assumption of the flow without separation we have $[1-3]$

$$
\begin{aligned}
& F_{x}=-\int_{0}^{2 \pi} p(r \cos \varphi ; r \sin \varphi) r \cos \varphi d \varphi \\
& F_{y}=-\int_{0}^{2 \pi} p(r \cos \varphi ; r \sin \varphi) r \sin \varphi d \varphi
\end{aligned}
$$

The integrand is defined by (24) with the substitution $x=r \cos \varphi, y=r \sin \varphi$.

Note that following pattern is detected. The terms of the form $x^{n} y^{m}$ with odd number $n+m$ give a non-zero contribution to right-hand sides of (25). For $F_{x}$ such terms are $x, x y^{2}, x^{3}$. For $F_{y}$ such terms are $y, x^{2} y, y^{3}$. Then relations (25) are taransformed to

$$
\begin{aligned}
& F_{x}=-\pi r^{2}\left(p_{10}+\frac{r^{2}}{4}\left(p_{12}+3 p_{30}\right)\right), \\
& F_{y}=\pi r^{2}\left[g-p_{01}-\frac{r^{2}}{4}\left(p_{21}+3 p_{03}\right)\right] .
\end{aligned}
$$

Coefficients $p_{n m}$ given in (26) should be calculated with the use of (8) and (11). Relations for $u$ and $v$ (Sec. 2.1) and relations for odd coefficients $b_{n m}$ (Sec. 2.2) should also be used. We obtain the following results

$$
\begin{aligned}
p_{10}=-a_{11}+ & 2 a_{02} a_{10}, \quad p_{01}=-a_{11} a_{10}+2\left(1+3 r^{2}\right) a_{02}+4 a_{10}+e_{1} r^{2} \frac{\left(3 r^{2}-2\right)}{\left(2-r^{2}\right)}, \\
3 p_{30}+p_{12}= & \frac{\left(7 r^{2}-24\right)}{4\left(2-r^{2}\right)} a_{11}^{2}+18 r^{2} a_{02}^{2}-\frac{10}{r^{2}} a_{10}^{2}-6 \frac{\left(r^{2}+2\right)}{r^{2}} a_{02} a_{10}+ \\
& +e_{1} \frac{\left(26-15 r^{2}\right)}{2\left(2-r^{2}\right)} a_{10}+\frac{10}{r^{2}\left(2-r^{2}\right)} a_{11}-9 e_{1} r^{2} a_{02}, \\
3 p_{03}+p_{21}= & -\frac{3 r^{2}\left(9+r^{2}\right)}{2\left(2-r^{2}\right)^{2}} a_{11} a_{02}+\frac{\left(-r^{4}-28 r^{2}+20\right)}{2 r^{2}\left(2-r^{2}\right)} a_{11} a_{10}+\frac{\left(75 r^{4}-72 r^{2}-24\right)}{r^{2}\left(2-r^{2}\right)} a_{02}+ \\
& +\frac{10\left(5 r^{2}-6\right)}{r^{2}\left(2-r^{2}\right)} a_{10}+e_{1} \frac{r^{2}\left(-30 r^{4}+91 r^{2}-90\right)}{4\left(2-r^{2}\right)^{2}} a_{11}+e_{1} \frac{\left(75 r^{4}-156 r^{2}+92\right)}{2\left(2-r^{2}\right)^{2}} .
\end{aligned}
$$

The right hand side of (27) contains still unknown coefficients $a_{11}, a_{02}, a_{10}$. To determine them one need to solve the system of nonlinear equations (23). The analytical solution of (23) is rather difficult. Let us consider the case $r^{2} \ll 1$. It means that the radius of the cylinder is small in comparison with the size of incoming flow. Let us construct a solution of (23) in the 
form of expansion in term of the small parameter $r^{2}$ [12]. Then unknown base coefficients are represented in the form

$$
a_{n m}=a_{n m}^{(0)}+r^{2} a_{n m}^{(1)}+r^{4} a_{n m}^{(2)}+r^{6} a_{n m}^{(3)}+\ldots
$$

Preliminary analysis shows that in our case it is necessary to set $a_{n m}^{(0)}=0$. Coefficients $a_{n m}^{(1)}$, $a_{n m}^{(2)}, a_{n m}^{(3)}$ are as follows

$$
\begin{aligned}
& a_{11}^{(1)}=0, \quad a_{02}^{(1)}=\frac{7 e_{1}}{8}, \quad a_{10}^{(1)}=\frac{3 e_{1}}{2}, \\
& a_{11}^{(2)}=-\frac{15 e_{1}^{2}}{2}, \quad a_{02}^{(2)}=-\frac{11 e_{1}}{16}, \quad a_{10}^{(2)}=-\frac{9 e_{1}}{4}, \\
& a_{11}^{(3)}=\frac{123 e_{1}^{2}}{8}, \quad a_{02}^{(3)}=\frac{45 e_{1}^{3}}{8}+\frac{53 e_{1}}{64}, \quad a_{10}^{(3)}=\frac{27 e_{1}}{16} .
\end{aligned}
$$

Transforming relations (26) and taking into account (28) and (29), we obtain relations for drag and lift

$$
\begin{aligned}
& F_{x}=\pi r^{2}\left[r^{4}\left(\frac{51}{8} e_{1}^{2}\right)+r^{6}\left(-\frac{45}{4} e_{1}^{2}\right)+\ldots\right], \\
& F_{y}=\pi r^{2}\left[g+r^{2}\left(\frac{17}{4} e_{1}\right)+r^{4}\left(-\frac{59}{8} e_{1}\right)+r^{6}\left(\frac{135}{16} e_{1}^{3}+\frac{117}{32} e_{1}\right)+\ldots\right] .
\end{aligned}
$$

Relations (30) are new. They allow us to calculate the values of $F_{x}$ and $F_{y}$ in relation to parameter $r$ and asymmetry parameter $e_{1}$.

\section{The D'Alembert's paradox}

The obtained above relations are convenient to analyze the D'Alembert's paradox under close to real conditions. Let us compare drag and lift values obtained according to (30).

$F_{x}$ depends on parameters $r$ and $e_{1}$. Whereas $F_{y}$ depends also on $g$. The latter is the contribution from the buoyancy force. We designate the buoyancy force as $F_{a r h}$. The dimensionless value of $F_{a r h}$ is

$$
F_{a r h}=\pi r^{2} \frac{9.8 L}{U_{0}^{2}} .
$$

The force $F_{\text {arh }}$ is directed vertically upward. Let us calculate the value of this force for some real flow. Suppose, for example, $L=10(m)$ and $U_{0}=20\left(\frac{m}{s e c}\right)$ then $\frac{F_{a r h}}{\pi r^{2}}=2.45 \cdot 10^{-1}$.

It seems logical to compare the value of $F_{a r h}$ and the value of actual lifting force caused by the incoming flow. We designate this force as $R_{y}$. It follows from (30) that

$$
F_{y}=F_{a r h}+R_{y} .
$$

Let us calculate the value of lifting force for some values of $r$ and $e_{1}$. Let consider $r=0.2$ and $r=0.6$. The asymmetry parameters are $e_{1}=0.1, e_{1}=1.0, e_{1}=2.0$. Calculated values of $\frac{R_{y}}{\pi r^{2}}$ are presented in Tab. 1 .

Let us compare the values of $\left|R_{y}\right|$ and $F_{\text {arh }}$ in the real flow for the given above values of parameters. The values of the ratio $\frac{\left|R_{y}\right|}{F_{\text {arh }}}$ are presented in Tab. 2. 
Table 1. Value of $\frac{R_{y}}{\pi r^{2}}$ in relation to $r$ and $e_{1}$

\begin{tabular}{|c|l|l|l|}
\hline$r \backslash e_{1}$ & \multicolumn{1}{|c|}{0.1} & \multicolumn{1}{c|}{1.0} & \multicolumn{1}{c|}{2.0} \\
\hline 0.2 & $1.58 \cdot 10^{-2}$ & $1.59 \cdot 10^{-1}$ & $3.21 \cdot 10^{-1}$ \\
\hline 0.6 & $7.49 \cdot 10^{-2}$ & 1.13 & 4.64 \\
\hline
\end{tabular}

Table 2. Value of $\frac{\left|R_{y}\right|}{F_{\text {arh }}}$ in relation to $r$ and $e_{1}$

\begin{tabular}{|c|l|l|l|}
\hline$r \backslash \backslash e_{1}$ & \multicolumn{1}{|c|}{0.1} & \multicolumn{1}{|c|}{1.0} & \multicolumn{1}{c|}{2.0} \\
\hline 0.2 & $6.47 \cdot 10^{-2}$ & $6.51 \cdot 10^{-1}$ & 1.31 \\
\hline 0.6 & $3.06 \cdot 10^{-1}$ & 4.65 & 18.93 \\
\hline
\end{tabular}

Tab. 2 shows that there is a range of values of $r$ and $e_{1}$ where $\left|R_{y}\right|$ is much smaller than $F_{a r h}$. This occurs, for example, when $r=0.2$ and $e_{1}=0.1$. In contrast, for $r=0.6$ and $e_{1}=2.0$ the value of $\left|R_{y}\right|$ far exceeds $F_{a r h}$ and the contribution of the buoyancy force in $F_{y}$ can be neglected.

Let us proceed to the analysis of the drag force $F_{x}$. In our case it is described by first relation (30). Comparison of obtained relations for $F_{x}$ and $R_{y}$ leads to the following conclusions.

1. The first terms in these relations have different orders with respect to $r^{2}$. The first term in relation for $F_{x}$ is proportional to $r^{6}$ while in relation for $R_{y}$ is proportional to $r^{4}$. It means that the value of the drag $F_{x}$ is far less than the magnitude of the force $R_{y}$ for small values of $r^{2}$. Since we assume that the value of $r^{2}$ is small then the assertion is true for our case. One should note that it is valid only for sufficiently small values of $e_{1}$.

2. The first terms in relations for $F_{x}$ and $R_{y}$ depend variously on parameter $e_{1}$. The first term in relation for $F_{x}$ is proportional to $e_{1}^{2}$ while the first term in relation for $R_{y}$ is proportional to $e_{1}$. If the asymmetry parameter is greater than 1 then the relation between $F_{x}$ and $F_{y}$ can be changed even for a small value of $r^{2}$. Then $F_{x}$ may be comparable to $R_{y}$ in magnitude.

Calculations confirm this analysis. The values of $\frac{F_{x}}{\pi r^{2}}$ calculated from (30) for various values of $r$ and $e_{1}$ are presented in Tab. 3 .

Table 3. Value of $\frac{F_{x}}{\pi r^{2}}$ in relation to $r$ and $e_{1}$

\begin{tabular}{|c|c|c|c|}
\hline$r \backslash \backslash e_{1}$ & 0.1 & 1.0 & \multicolumn{1}{c|}{2.0} \\
\hline 0.2 & $9.48 \cdot 10^{-5}$ & $9.48 \cdot 10^{-3}$ & $3.79 \cdot 10^{-2}$ \\
\hline 0.6 & $3.01 \cdot 10^{-3}$ & $3.01 \cdot 10^{-1}$ & 1.21 \\
\hline
\end{tabular}

Let us compare the values of $F_{x}$ and $R_{y}$ presented in Tabs. 3 and 1 for the same values of parameters. It is evident that situation characteristic of the D'Alembert's paradox takes place. The value of $F_{x}$ is far less than $F_{y}$. This is true regardless of whether or not the contribution from the buoyancy force in (32) is included. For example, if $r=0.2$ and $e_{1}=0.1$ we have 
$\frac{F_{x}}{\pi r^{2}}=9.48 \cdot 10^{-5}, \frac{R_{y}}{\pi r^{2}}=1.58 \cdot 10^{-2}, \frac{F_{x}}{R_{y}}=6.0 \cdot 10^{-3}$. So $F_{x}$ is far less than $R_{y}$. However, when parameters are close to $r=0.6$ and $e_{1}=2.0$ this trend is violated. In this case $\frac{F_{x}}{\pi r^{2}}=1.21$ and $\frac{F_{y}}{\pi r^{2}}=4.64$ (see Tabs. 3 and 1 ) and the drag and the lift are already comparable in magnitude, so as $\frac{F_{x}}{R_{y}}=0.26$. The D'Alembert's paradox in its classic version does not arise. The drag is less than lift but they are comparable in magnitude.

Analyzing relations (30), (32), one can find another case when the D'Alembert's paradox does not take place. This case corresponds to the negative value of the asymmetry parameter

$$
e_{1}<0
$$

In this case the ratio between horizontal and vertical components of the force acting on the streamlined body is changed. In fact, if inequality (33) is satisfied then the sign of $F_{x}$ is not changed because $F_{x}$ is proportional to $e_{1}^{2}$, according to the first relation (30). In contrast, the sign of $R_{y}$ is reversed, because $R_{y}$ is proportional to $e_{1}$. Since the buoyancy force $F_{\text {arh }}$ is always positive the magnitude of $F_{y}$ is decreased. This means that the ratio $\frac{F_{x}}{F_{y}}$ is also decreased. Then $F_{x}$ and $F_{y}$ are comparable in magnitude and situation characteristic of the D'Alembert's paradox does not arise.

Let us consider one example. Let $r=0.2$, and $e_{1}=-1$. As it follows from Sec. 2.1 this corresponds to the initial longitudinal velocity profile

$$
u(-1, y)=1-y+e_{2} y^{2}-1.042 y^{3}+e_{4} y^{4},
$$

where $e_{2}$ and $e_{4}$ have arbitrary values.

The values of $e_{2}$ and $e_{4}$ may be well over the value of $e_{1}$. For example, let us assume that $e_{2}=10^{3}$ and $e_{4}=10^{3}$. Then we have slightly asymmetrical velocity profile.

Suppose that the values of $L$ and $U_{0}$ are identical to those used in previous calculations. Then, as shown in Tab. 2, $\frac{\left|R_{y}\right|}{F_{\text {arh }}}=6.51 \cdot 10^{-1}$. In this case $R_{y}=-0.651 \cdot F_{\text {arh }}$. According to (32), we have $F_{y}=0.349 \cdot F_{\text {arh }}=8.55 \cdot 10^{-2}$. As it is shown in Tab. 3 , we have $F_{x}=9.48 \cdot 10^{-3}$ for $e_{1}=1$. The same result is obtained for $e_{1}=-1$ and hence, $\frac{F_{x}}{F_{y}}=1.11 \cdot 10^{-1}$. The ratio of the drag and the lift is of the order of $10^{-1}$, and the D'Alembert's paradox is not observed.

\section{Conclusion}

The results of the study are as follows. When considering the D'Alembert paradox under real conditions we assume that the incoming flow is symmetrical, the ratio of the streamlined body size to the size of the incoming flow is small, and the extent of the flow is infinite in both directions. If these assumptions are modified or relaxed then the D'Alembert's paradox may not occur in its classic version.

The D'Alembert's paradox may also not occur if the influence of the gravity force is taken into account. 


\title{
References
}

[1] L.I.Sedov, Continuum Mechanics, Part 2, Moscow, Nauka, 1970 (in Russian).

[2] N.E.Kochin, I.A.Kibel, N.V.Rose, Theoretical Hydromechanics, Part 1, Moscow, Fismatlit, 1963 (in Russian).

[3] S.V.Vallander, Lectures on Hydroaeromechanics, Leningrad Gos. Univ., Leningrad, 1978 (in Russian).

[4] G.K.Batchelor, Introduction to Fluid Dynamics, Cambridge Univsrsity Press, 1967.

[5] G.Grinberg, W.Pauls, U.Frish, Genesis of d'Alembert's paradox and analitical elaboration of the drag problem, Physica D, 237(2008), 1878-1886.

[6] L.D.Landau, E.M.Lifshits, The Course of Theoretical Physics. Fluid Mechanics, Moscow, Nauka, 1986 (in Russian).

[7] A.V.Koptev, Integrals of Navier-Stokes equations, Saransk. Trudi Srednevolzhskogo matematicheskogo obshchestva, 6(2004), no. 1, 215-225 (in Russian).

[8] A.V.Koptev, First integral and ways of further integration of Navie-Stokes equations, Izv. Ros. gos. pedagog. univ. A. I. Gertsena, Saint-Petersburg, (2012), no. 147, 7-17 (in Russian).

[9] A.V.Koptev, First integral of equations for icompressible fluid flow, Proceedings of 11-th AllRussian Congress on fundamental problems of theoretical and applied mechanics, Kazan, Povolzhskiy federalniy universitet, 2015, 1957-1959 (in Russian).

[10] A.V.Koptev, Generator of Solution of 2D Navier-Stokes Equations, Journal of Siberian Federal University. Math. and Phys., 7,(2014), no. 3, 324-330.

[11] A.V.Koptev, Structure of Solution of the Navier-Stokes Equations, Vestnik natsionalnogo issledovatelskogo yadernogo universiteta MEPI, 3,(2014), no. 6, 656-660.

[12] A.H.Nayfeh, Perturbation Methods, John Wiley \& Sons, Inc., 1973.

\section{Парадокс Даламбера в условиях, приближенных к реальным}

\author{
Александр В. Коптев \\ Институт водного транспорта \\ Государственный университет морского и речного флота им. адм. С. О. Макарова \\ Двинская, 5/7, Санкт-Петербург, 198035 \\ Россия
}

В статъе представлено исследование парадокса Даламбера при обтекании иилиндра потоком идеальной несжимаемой среды при условиях, приближенных $к$ реальным. Профиль скорости набегающего потока задан в некотором сечении на конечном расстоянии от обтекаемого тела. Введен дополнительный параметр, определяющий степень асимметричности набегающего потока. $B$ зависимости от значения этого параметра, который изначально предполагается малым, и в зависимости от параметра, определяющего геометрические размеры обтекаемого тела, выделены случаи, когда ситуация близка к парадоксу Даламбера в его классическом варианте, а когда нет.

Ключевые слова: идеальная несжимаемая среда, обтекание иилиндра, уравнения Эйлера, интеграл, профиль скорости, асимметричность потока, подвемная сила, лобовое сопротивление. 